\title{
Zur Kenntnis der komplexen anorganischen Säuren.
}

\author{
Von \\ F. Kehrmans. \\ VIII. Abhandlung.
}

Über Arsenduodeciwolframsäure, Arsenluteowolframsäure und ihre Spaltungsprodukte.
Nach gemeinsam mit Herrn E. RÜtтIMaNN ausgeführten Versuchen.

In der letzten Mitteilung über komplexe Säuren ${ }^{1}$ hat der eine von uns erwähnt, dals unter Einhaltung bestimmter Versuchsbedingungen die Darstellung eines Ammonsalzes der Arsenduodeciwolframsäure gelingt.

Wir haben diese Beobachtung weiter verfolgt, das betr. Ammonsalz analysiert und dessen vermittelst Alkalikarbonaten entstehendes Spaltungsprodukt studiert. Dabei hat sich ergeben, dals die Arsenduodeciwolframate existenzfähig, und, abgesehen von geringerer Beständigkeit, namentlich der freien Säure, den Phosphorduodeciwolframaten in jeder Beziehung analog sind. Ferner hat das erneute Studium der durch Kochen von Natriumwolframat mit syrupdicker Arsensäure entstehenden Luteowolframate, wie bereits früher angedeutet $^{2}$ die Formel $3 \mathrm{R}_{2} \mathrm{O} \cdot \mathrm{As}_{2} \mathrm{O}_{5} .18 \mathrm{WO}_{3}+$ aq. mit aller Sicherheit festgestellt, und ferner ergeben, dafs die Spaltung dieser Salzreihe durch Alkalikarbonate genau ebenso verläuft, wie die früher studierte analoge Zersetzung der Phosphorluteowolframate und Phosphorluteomolybdate. Hiermit sind die letzten Unsicherheiten auf diesem Gebiete bezüglich der Zusammensetzung einiger der Salzreihen, welche

\footnotetext{
1 Z. anorg. Chem. 7, 420 unten.

2 1. c. S. 408 .
} 
von mir und meinen Mitarbeitern untersucht worden sind, beseitigt. Wir betrachten daher die Versuche über diejenigen komplexen Säuren und Salzreihen, welche einerseits aus Phosphorsäure und Arsensäure, andererseits aus Wolframsäure und Molybdänsäure erhalten wurden, als vorläufig abgeschlossen und beabsichtigen nunmehr die Siliko- und Borowolframate, welche von MarignaC und von KLEIN eingehend erforscht zu sein scheinen, etwas näher ins Auge zu fassen.

\section{Experimenteller Teil.}

I. Über Arsenduodeciwolframsäure.

Nach einer Reihe von orientierenden Versuchen zur Darstellung des früher erwähnten Ammonsalzes sind wir zum folgenden Verfahren gelangt, welches bezüglich Ausbeute und Reinheit sehr befriedigende Resultate liefert.

Eine konzentrierte wässerige Lösung von $8 \mathrm{Mol} . \mathrm{Na}_{2} \cdot \mathrm{WO}_{4}+\mathrm{aq}$ wird bei gewöhnlicher Temperatur mit $1 \mathrm{Mol}$. Arsensäure $\mathrm{H}_{3} \mathrm{AsO}_{4}$ vermischt. Hierzu fügt man tropfenweise unter Umrühren verdünnte Salzsäure, wobei sich der momentan entstehende Niederschlag von Wolframsäurehydrat schnell löst. Man fährt mit dem Zusatz der Salzsäure fort bis zum Eintritt stark saurer Reaktion, vermischt die abgekühlte Flüssigkeit, eventuell nach vorheriger Filtration mit ziemlich viel reinem, festem Chlorammonium und erhitzt unter Umschütteln langsam bis zum Sieden. Nach kurzer Zeit trübt sich die anfangs klare Flüssigkeit und scheidet bald einen dichten schweren weilsen krystallinischen Niederschlag ab. Man läst absitzen, entfernt die darüber stehende Flüssigkeit durch Dekantation und wäscht zunächst wiederholt mit einer freie Salzsäure enthaltenden wässerigen Salmiaklösung, ebenfalls unter Dekantation aus. Dann bringt man den Niederschlag auf ein Saugfilter und wäscht mit kleinen Mengen Wasser möglichst vollständig aus. Das so dargestellte Salz bildet ein blendend weilses, schweres mikrokrystallinisches Pulver vom Aussehen der analogen Phosphorverbindung. Von dieser unterscheidet es sich hauptsächlich durch seine bedeutendere Wasserlöslichkeit, worauf man beim Auswaschen Rücksicht zu nehmen hat. Zur Analyse wurde es im Exiccator über Schwefelsäure bis zu konstantem Gewicht getrocknet. Letztere wurde in der Weise ausgeführt, dals in getrennten Portionen: erstens durch Erhitzen auf Rotglut bis zu konstantem Gewicht die Wolframsäure, zweitens 
durch Auflösen in Natronlauge in einer Platinschale, Versetzen mit genügend Chlorammonium und doppelte Fällung mit Magnesiamischung die Arsensäure und endlich durch Destillation mit Natronlauge und Titration das Ammoniak bestimmt wurde. Das Wasser ergab sich alsdann aus dem Glühverlust minus Ammoniak und Arsensäure. Es zeigt sich, dals die geglühte $\mathrm{WO}_{3}$ nur eine Spur Arsensäure enthielt. Dieselbe wurde bestimmt und in Rechnung gezogen, ohne indessen das Resultat wesentlich zu beeinflussen.

\section{A. Bestimmung}

des A tomverbältnisses der beiden Säureanhydride.

I. $1.5682 \mathrm{~g}$ wasserhaltiges Ammonsalz gaben nach dem Glühen

$1.4122 \mathrm{~g} \mathrm{WO}_{3}$,

$2.6102 \mathrm{~g}$ desselben Salzes gaben $0.1340 \mathrm{Mg}_{2} \mathrm{As}_{2} \mathrm{O}_{7}$ entsprechend

$0.0994 \mathrm{~g} \mathrm{As}_{2} \mathrm{O}_{5}$.

II. $1.6294 \mathrm{~g}$ wasserh. Salz gaben $1.4624 \mathrm{WO}_{\mathrm{g}}$, $3.0752 \mathrm{~g}$ desselben $\quad 0.1552 \mathrm{Mg}_{2} \mathrm{As}_{2} \mathrm{O}_{7}$, entspr. $0.1151 \mathrm{As}_{2} \mathrm{O}_{5}$.

III. $2.0558 \mathrm{~g}$ wasserh. Salz $" 1.8500 \mathrm{WO}_{3}+\mathrm{As}_{2} \mathrm{O}_{5}$. Hierin wurden

$0.0078 \mathrm{~g} \mathrm{Mg}_{2} \mathrm{As}_{2} \mathrm{O}_{7}$ entspr. $0.0057 \mathrm{As}_{2} \mathrm{O}_{5}$ und

$1.8443 \mathrm{~g} \mathrm{WO}_{3}$ gefunden,

$3.2698 \mathrm{~g}$ wasserh. Salz gaben $0.1652 \mathrm{Mgg}_{2} \mathrm{As}_{2} \mathrm{O}_{7}$, entspr. $0.1225 \mathrm{As}_{2} \mathrm{O}_{5}$.

Hieraus berechnet sich

$$
\begin{aligned}
& \text { aus Analyse I. } \mathrm{As}_{2} \mathrm{O}_{5}: \mathrm{WO}_{3}=1: 23.49 \text {, } \\
& " \quad, \quad \text { II. } ", \quad=1: 24 \text {. } \\
& " \quad ", \text { III. } ", \quad "=1: 23.61 \text {, }
\end{aligned}
$$

was einem Verhältnis von $1 \quad \mathrm{As}_{2} \mathrm{O}_{\tilde{3}}: 24 \mathrm{WO}_{3}$ genügend genau entspricht.

$$
\begin{aligned}
& \text { B. Bestimmung des Ammoniaks. } \\
& \text { l. } 2.2974 \mathrm{~g} \text { gaben } 0.03673 \mathrm{NH}_{3}=1.60 \% \\
& \text { II. } 1.8192 \mathrm{~g} " \quad 0.03050 \mathrm{NH}_{3}=1.62 \%
\end{aligned}
$$

Berechnet für $3\left(\mathrm{NH}_{4}\right)_{2} \mathrm{O} . \mathrm{As}_{2} \mathrm{O}_{5} .24 \mathrm{WO}_{3}+12 \mathrm{H}_{2} \mathrm{O}: \mathrm{NH}_{3}=1.65 \%$.

Das beschriebene Ammoniumsalz entspricht also in Eigenschaften und Zusammensetzung der bisher unbekannten Arsenduodeciwolframsäure.

Wir haben natürlich den Versuch gemacht, diese daraus darzustellen und zwar nach der Methode, welche Debrax mit Erfolg zur Darstellung der Phosphorduodecimolybdänsäure aus ihrem Ammonsalz benutzt hat.

In der That löste sich das arsenduodeciwolframsaure Ammon durch Erwärmen mit Königswasser auf dem Wasserbade nach einiger Zeit teilweise, aber nie rollständig, auch nicht bei länger fortgesetzter Einwirkung, auf. Die von dem Ungelösten abfiltrierte Flüssigkeit 
enthielt zwreifelsohne die freie Duodecisäure in ziemlicher Menge, da sie mit Chlorammonium sofort das unlösliche Ammonsalz abschied, jedoch ist es uns leider noch nicht gelungen, die Säure krystallisiert zu erhalten, da sich die Lösung beim Eindampfen unter Ausscheidung von Wolframsäurehydrat total zersetzte. Auch die Darstellung des Baryum-, Kalium- und Natriumsalzes ist uns bisher nicht befriedigend geglückt, obwohl wir Andeutungen von deren Existensfähigkeit erbalten haben.

\section{Spaltung der Arsenduodeciwolframsäure durch Ammonkarbonat.}

Um direkte Beweise für die vorausgesetzte Analogie des beschriebenen Ammonsalzes mit phosphorduodeciwolframsaurem Ammon zu erhalten, wurde die Einwirkung von Ammonkarbonat auf dasselbe untersucht. Dieselbe verläuft nun genau entsprechend der Einwirkung desselben Salzes auf die genannte Phosphorverbindung. Es entstehen Salze der Reihe: $7 \mathrm{R}_{2} \mathrm{O} \cdot \mathrm{As}_{2} \mathrm{O}_{5} \cdot 22 \mathrm{WO}_{3}+\mathrm{aq}$, in jeder Beziehung ähnlich und vielfach isomorph mit den früher ${ }^{1}$ beschriebenen Salzen $7 \mathrm{R}_{2} 0 . \mathrm{P}_{2} \mathrm{O}_{5} \cdot 22 \mathrm{WO}_{3}+\mathrm{aq}$.

Überschüssige Mineralsäuren zerstören leicht das Molekül dieser in wässeriger Lösung recht beständigen Salze, indem neue Verbindungen entstehen, unter denselben wahrscheinlich Salze der Säure $3 \mathrm{H}_{2} \mathrm{O} . \mathrm{As}_{2} \mathrm{O}_{5} .21 \mathrm{WO}_{3}+\mathrm{aq}$, jedoch konnten diese Substanzen in Anbetracht ihrer grofsen Veränderlichkeit beim Umkrystallisieren bisher nicht befriedigend charakterisiert werden.

$20 \mathrm{~g}$ Duodecisalz wurden in $100 \mathrm{ccm}$ Wasser suspendiert, zum gelinden Sieden erhitzt und tropfenweise eine $5 \%$ ige Lösung von Ammonkarbonat binzugefügt, bis der Niederschlag bis auf einen kleinen Rest gelöst war. Hierauf wurden $5 \mathrm{~g}$ krystallisiertes Chlorbaryum hinzugefügt, von dem unbedeutenden flockigen Niederschlag von Baryumwolframat abfiltriert und das wasserklare Filtrat abgekühlt. In demselben bildete sich schnell eine reichliche Krystallisation des neuen Baryumsalzes in Form grofser, aus kleinen glänzendweifsen Oktaëdern zusammengesetzter Doppelpyramiden. Um diese vollständig von dem darin in isomorpher Mischung enthaltenden Ammonsalz zu befreien, warde sie viermal wiederholt in heirsem Wasser gelöst und mit festem $\mathrm{BaCl}_{2}$ ausgesalzen. Schlielslich wurde das Salz nochmals, nach vorheriger Entfernung der Mutterlauge, aus

1 Ber. deutsch. chem. Ges. 24, 2334. 
heifsem Wasser umkrystallisiert, abgesaugt und bis zum Verschwinden der Chlorreaktion mit kleinen Quantitäten kalten Wassers ausgewaschen. Ein so dargestelltes Präparat diente in lufttrockenem Zustande zur Analyse.

Die Baryumbestimmung konnte in der früher beschriebenen Weise ${ }^{1}$ durch Schmelzen mit kohlensaurem Natronkali und Wiegen als Sulfat mit sehr befriedigenden Resultat ausgeführt werden; dagegen gelang es nicht, in der von $\mathrm{BaCO}_{3}$ abfiltrierten alkalischen Lösung die Arsensäure zu bestimmen, da sich das Arsen während des Schmelzens teilweise mit dem Tiegel legierte, teilweise verHiüchtigte, so dafs viel zu wenig erhalten wurde. Es wurde daher ein anderer Weg eingeschlagen. Aus der wässerigen Lösung des Ba-Salzes wurde mittels verdünnter Schwefelsäure das Ba möglichst genau ausgefällt und eventuell nach vorherigem Verschmelzen mit kohlensaurem Natronkali als Sulfat bestimmt. Das die Zersetzungsprodukte der 22 Arsenwolframsäure enthaltende Filtrat wurde in einer Platinschale zur Trockne verdampft, der Rückstand mit Natronlauge in Lösung gebracht und darin das Arsen in bekannter Weise als Pyroarseniat bestimmt. So wurden folgende Resultate erhalten:

I. $4.4010 \mathrm{~g}$ wasserhaltiges Salz gaben durch Erhitzen $3.8209 \mathrm{~g}$ Rückstand. Hierin fanden sich $0.63406 \mathrm{~g} \mathrm{BaO}$.

II. $4.6932 \mathrm{~g}$ gaben $4.0724 \mathrm{~g}$ Rüickstand und darin $0.6816 \mathrm{BaO}$.

III. $4.3274 \mathrm{~g} \quad, \quad 3.7549 \mathrm{~g} \quad$ ",$\quad$ " $0.6229 \quad$,

IV. $5.0610 \mathrm{~g}, \quad 4.3927 \mathrm{~g} \quad " \quad " \quad, 0.7279 \quad "$

V. $5.5978 \mathrm{~g} \quad " 4.8613 \mathrm{~g} \quad " \quad " \quad, 0.8097 \quad "$

VI. $3.7936 \mathrm{~g}$ wasserhaltiges Salz, entsprechend 3.2971 wasserfreies gaben $0.11706 \quad \mathrm{As}_{2} \mathrm{O}_{5}$.

VII. $4.5522 \mathrm{~g}$ wasserh. Salz entspr. 3.9497 wasserfreies gaben $0.14089 \mathrm{As}_{2} \mathrm{O}_{5}$.

Berechnet für

Gefunden:

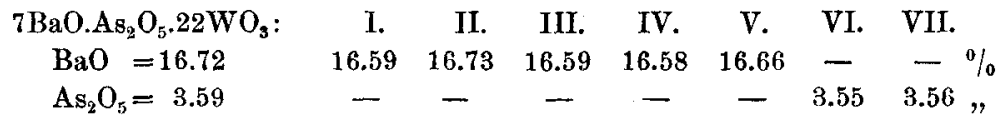

Berechnet für.

Gefunden:

die gleiche Formel mit 54 aq: I. II. III. IV. V. $\mathrm{H}_{2} \mathrm{O}=13.17 \% \quad 13.18 \quad 13.24 \quad 13.22 \quad 13.20 \quad 13.15 \%$

Durch doppelte Umsetzung des Baryumsalzes mit Sulfaten lassen sich noch eine Anzahl Salze dieser Reihe erhalten, welche jedoch nicht eingehender untersucht wurden. Dagegen schien es interessant,

1. c. S. 2328.

z. anorg. Chem. XXIr. 
nachzusehen, ob die Salze der 22. Reihe durch Mineralsäuren in gleicher Weise verändert würden, wie die analogen Phosphorverbindungen.

Zu diesem Zwecke wurde eine Quantität Ammonsalz der Duodecisäure zunächst durch Behandlung und Ammonkarbonat nach dem beschriebenen Verfahren in das Ammonsalz mit $22 \mathrm{WO}_{3}$ verwandelt und dessen Lösung bis zur deutlich sauren Reaktion mit verdünnter Salzsäure versetzt. Durch Erhitzen zum Sieden scheidet sich nunmehr in der That ein geringer Niederschlag von schwerlöslichem Duodecisalz aus und die davon abfiltrierte Flüssigkeit ergiebt auf Zusatz von festem Chlorammonium einen reichlichen weifsen, krystallinischen, in reinem Wasser sehr leicht löslichen Niederschlag. Leider zersetzte sich dieses Salz, wahrscheinlich die Verbindung $3\left(\mathrm{NH}_{4}\right)_{2} \mathrm{O} . \mathrm{As}_{2} \mathrm{O}_{5} .21 \mathrm{WO}_{3}$, jedesmal weitgehend bei wiederholten Versuchen, dieselbe durch Umkrystallisieren zu reinigen, so dals wir notgedrungen auf deren Studium verzichten mufsten.

\section{III. Über Arsenluteowolframsäure.}

Diese Verbindung, sowie eine Anzahl von Salzen derselben, sind zuerst von M. FREMERY ${ }^{1}$ erhalten und beschrieben, jedoch ganz unrichtig analysiert worden. Bald darauf ${ }^{2}$ hat der eine von uns diese Arbeit mit Rücksicht auf seine Voraussetzung der Analogie der FrEMERY'schen Arsenwolframsäure mit der von ihm selbst entdeckten und untersuchten Phosphorluteowolframsäure wiederholt, seine Vermutung bestätigt gefunden und auf Grund von, wie sich später herausstellte, noch immer nicht genügend genauen Analysen, für beide analogen Säuren die Formeln $3 \mathrm{H}_{2} \mathrm{O}_{2} \mathrm{P}_{2} \mathrm{O}_{5} \cdot 16 \mathrm{WO}_{3}+\mathrm{aq}$ und $3 \mathrm{H}_{2} \mathrm{O} \cdot \mathrm{As}_{2} \mathrm{O}_{5} \cdot 16 \mathrm{WO}_{3}+\mathrm{aq}$ aufgestellt.

Nachdem nun vor einigen Jahren ${ }^{3}$ die zweite Bearbeitung der Phosphorluteowolframate, gestützt auf sehr exakte analytische Methoden mit Sicherheit zu der Formel $3 \mathrm{H}_{2} \mathrm{O}_{2} \mathrm{P}_{2} \mathrm{O}_{5} \cdot 18 \mathrm{WO}_{3}+\mathrm{aq}$ der Säure resp. $3 \mathrm{R}_{2} 0 . \mathrm{P}_{2} \mathrm{O}_{5} \cdot 18 \mathrm{WO}_{3}+\mathrm{aq}$ der Salze gefïhrt hatte, und sich gleichzeitig gezeigt hatte, dafs die Einwirkung von Alkalikarbonaten eine spaltende, und nicht, wie früher angenommen, neutralisierende ist, mufste die Arsenluteowolframsäure zum dritten Male studiert werden, um auch hier mit Hilfe der inzwischen gewonnenen analyti-

\footnotetext{
1 Doktor-Dissertation (Freiburg i/B. 1884).

${ }^{2}$ Lieb. Ann. 245, 49.

${ }^{8}$ Z. anorg. Chem. 4, 138.
} 
schen Erfahrung die früher aufgestellte, wahrscheinlich unrichtige Formel zu kontrollieren. Die nachstehend beschriebenen Versuche haben die Frage im vermuteten Sinne zur bestimmten Entscheidung gebracht. Die Arsenluteowolframsäure besitzt die Zusammensetzung , $3 \mathrm{H}_{2} \mathrm{O} . \mathrm{As}_{2} \mathrm{O}_{5} .18 \mathrm{WO}_{3}+\mathrm{aq}^{4 *}$ und ihre Spaltung durch Karbonate verläuft nach der Gleichung:

$$
3 \mathrm{~K}_{2} \mathrm{O}_{2} \cdot \mathrm{As}_{2} \mathrm{O}_{5} .18 \mathrm{WO}_{3}+3 \mathrm{~K}_{2} \mathrm{O}=5 \mathrm{~K}_{2} \mathrm{O} \cdot \mathrm{As}_{2} \mathrm{O}_{5} .17 \mathrm{WO}_{3}+\mathrm{K}_{2} \mathrm{WO}_{4} \text {. }
$$

Es besteht also bis ins Einzelne vollkommen Analogie mit der Phosphorluteowolframsäure.

Wir haben uns die zu den Versuchen nötige Arsenluteowolframsäure anfangs nach den Angaben ihres Entdeckers Fremery dargestellt. Da dieselbe aus uns unbekannt gebliebenen Ursachen bisweilen mifslang, haben wir nach einer zuverlässigeren Methode gesucht und dieselbe in der Einwirkung überschüssiger konzentrierter Arsensäurelösung auf gewöhnliches Natriumwolframiat gefunden, d. i. ganz entsprechend der Darstellung der Phosphorluteowolframsäure aus demselben Salz und wässeriger Phosphorsäure.

$100 \mathrm{~g}$ krystallisiertes Natriumwolframiat wurden in der gerade ausreichenden Menge siedenden Wassers gelöst, portionsweise mit syrupdicker Arsensäurelösung versetzt, bis die Flüssigkeit stark saure Reaktion und gelbliche Farbe zeigt und nun nach nochmaligem Zusatz von etwa halb soviel Arsensäure als bereits vorhanden, so lange unter Erneuerung des verdampfenden Wassers zum Sieden erhitzt, bis die Intensität der bald entstehenden citronengelben Farbe sich nicht weiter veränderte. Darauf wurde mit festem Chlorammonium das Ammonsalz so gat wie vollständig ausgesalzen, abgesaugt, in reinem Wasser gelöst und das Aussalzen und Wiederauflösen im Ganzen vier Mal wiederholt. Schliefslich wurde der Krystallbrei in der eben ausreichenden Menge Wasser heifs gelöst filtriert, erkalten gelassen und die ausgeschiedenen Krystalle nochmals aus wenig heifsem Wasser umkrystallisiert.

So wurden dicke trikline citronengelbe prismatische Krystalle erhalten, welche in kaltem Wasser sehr löslich sind und dem Resultat der Analyse zufolge die Formel:

besitzen.

$$
3\left(\mathrm{NH}_{4}\right)_{2} \mathrm{O} . \mathrm{As}_{2} \mathrm{O}_{5} .18 \mathrm{WO}_{3}+14 \mathrm{aq}
$$

Die Bestimmung von Wolframsäure und Arsensäure, sowie von Ammoniak wurde in der beim arsenduodeciwolframsaurem Ammon beschriebenen Art ausgeführt. Um die arsensaure Ammonmagnesia 
zur Wägung zu bringen, wurde der doppelt gefällte Niederschlag nach dem Auswaschen auf dem Filter in ganz verdünnter Salpetersäure gelöst, die Lösung in einer Platinschale zur Trockne verdampft, der Rückstand geglüht und als Magnesiumpyroarseniat gewogen. So wurden folgende Resultate erhalten:

I. $1.7802 \mathrm{~g}$ wasserhaltiges Salz gaben nach dem Glühen

$1.5732 \mathrm{~g}$ Rückstand, in welchem $1.5517 \mathrm{~g} \mathrm{WO}_{\mathrm{s}}$ gefunden wurden.

$2.9278 \mathrm{~g}$ desselben Salzes, gleichzeitig abgewogen, gaben

$0.1457 \mathrm{~g} \mathrm{As}_{2} \mathrm{O}_{5}$.

II. $1.3378 \mathrm{~g}$ wasserh. Salz gaben $1.1814 \mathrm{~g}$ Rückstand und $1.1691 \mathrm{WO}_{3}$,

$1.7810 \mathrm{~g}$ desselben " $"$ gleichzeitig abgewogen

$0.8814 \mathrm{~g} \mathrm{As}_{2} \mathrm{O}_{5}$.

Hieraus folgt aus I. das Atomverhältnis $\mathrm{As}_{2} \mathrm{O}_{5}: \mathrm{WO}_{3}=1: 17.37$, "II. " " " $\quad \mathrm{As}_{2} \mathrm{O}_{5}: \mathrm{WO}_{\mathrm{s}}=1: 17.66$,

Zahlen, welche entschieden für das Verhältnis $\mathrm{As}_{2} \mathrm{O}_{5}: W \mathrm{O}_{3}=1: 18$

sprechen. Dasselbe ergiebt sich indessen etwas genauer aus der Analyse der freien Säure.

III. $1.5622 \mathrm{~g}$ wasserhaltiges Salz gaben $0.03248 \mathrm{NH}_{3}$.

IV. $0.7422, \quad, \quad, \quad, \quad 0.01538 \mathrm{NH}_{3}$.

\begin{tabular}{ccc} 
Berechnet für & \multicolumn{2}{c}{ Gefunden: } \\
$3\left(\mathrm{NH}_{4}\right)_{2} \mathrm{O} . \mathrm{As}_{2} \mathrm{O}_{5} .18 \mathrm{WO}_{3}+14 \mathrm{H}_{2} \mathrm{O}:$ & $\mathrm{I}$. & II. \\
$\mathrm{NH}_{3}=2.12$ & $\mathrm{NH}_{\mathrm{B}}=2.07$ & $2.07 \%$.
\end{tabular}

Um zunächst das Verhältnis von $\mathrm{As}_{2} \mathrm{O}_{5}: \mathrm{WO}_{3}$ womöglich noch genauer festzustellen, wurde die freie Säure dargestellt und analysiert. $\mathrm{Zu}$ diesem Zwecke wurden $50 \mathrm{~g}$ Ammoniumsalz mit Königswasser auf dem Wasserbade so lange erwärmt, bis das Aufhören der Gasentwickelung die Zerstörung des Ammoniaks anzeigte und so oft wiederholt nach erneutem Wasserzusatz zur Trockne verdampft, bis sämtliche flüchtige Säure entfernt war. Der krystallinische Rückstand wurde dann zwei Mal aus ganz wenig Wasser umkrystallisiert. So wurden aus sechsseitigen Täfelchen bestehende citronengelbe, in Wasser äufserst lösliche Krystallkrusten erhalten, welche in lufttrockenem Zustande analysiert wurden und zwar nach der gleichen Methode wie das Ammoniumsalz.

I. $0.9938 \mathrm{~g}$ gaben $0.8235 \mathrm{~g} \mathrm{WO}$,

$1.8878 \mathrm{~g}$ gleichzeitig abgewogen gaben $0.0881 \mathrm{As}_{2} \mathrm{O}_{5}$.

II. $1.3674 \mathrm{~g}$ gaben $1.1246 \mathrm{~g} \mathrm{WO}$,

$2.4942 \mathrm{~g}$ gleichzeitig abgewogen gaben $0.1165 \mathrm{As}_{2} \mathrm{O}_{5}$.

Aus Analyse I folgt $\mathrm{H}_{3} \mathrm{AsO}_{4}: \mathrm{WO}_{3}: 1: 8.82$,

$" \quad$ II $, \mathrm{H}_{3} \mathrm{AsO}_{4}: \mathrm{WO}_{3}: 1: 8.77$,

also ziemlich genau $\mathrm{H}_{3} \mathrm{AsO}_{4}: \mathrm{WO}_{3}: 1: 9$. 
Um endlich noch in einem Falle das Verhältnis zwischen Arsensäure und Base möglichst genau festzustellen, wurde das Kaliumsalz dargestellt und analysiert. Die wässerige Lösung der freien Säure oder des Ammonsalzes wurde vier Mal wiederholt mit Chlorkalium ausgesalzen und das alsdann von Ammoniakgehalt befreite Kaliumsalz zwei Mal aus siedendem Wasser umkrystallisiert. Dasselbe ist bereits früher ${ }^{1}$ beschrieben und seine Isomorphie mit dem phosphorluteowolframsauren Kalium durch C. StuhLmann bewiesen worden.

Die Analyse wurde nach der früher ${ }^{2}$ genau beschriebenen Methode mit Hilfe von Chinolinchlorhydrat ausgeführt und das Kalium als Chlorid gewogen. Die Bestimmung der Arsensäure und des Krystallwassers konnte in diesem Falle in ein ond derselben Portion durch gelindes Glühen bis zur Gewichtskonstanz, Behandeln des Rückstandes mit Natronlauge und Fällen mit Magnesiamixtur ausgeführt werden, wobei die Arsensäure als Magnesiumpyroarseniat zur Wägung kam.

I. $4.7901 \mathrm{~g}$ wasserhaltiges Salz gaben 4.5328 Glührückstand, in welehem $0.2274 \mathrm{As}_{2} \mathrm{O}_{5}$ gefunden wurden.

II. $4.2738 \mathrm{~g}$ gaben 4.0416 Glührückstand und $0.20314 \mathrm{As}_{2} \mathrm{O}_{5}$.

III. $2.7206 \mathrm{~g}$ wasserhaltiges Salz, entsprechend $2.5755 \mathrm{~g}$ wasserfreiem, gaben $0.2372 \mathrm{~g} \mathrm{KCl}$, entsprechend $0.14965 \mathrm{~K}_{2} \mathrm{O}$.

IV. $2.7940 \mathrm{~g}$ entsprechend 2.6427 wasserfrriem, gaben $0.1616 \mathrm{~K}_{2} \mathrm{O}$.

\begin{tabular}{ccccc} 
Berechnet für die Formel & \multicolumn{4}{c}{ Gefunden: } \\
$3 \mathrm{~K}_{2} \mathrm{O} . \mathrm{As}_{2} \mathrm{O}_{5} .18 \mathrm{WO}_{3}+14 \mathrm{H}_{2} \mathrm{O}:$ & I. & II. & III. & IV. \\
$\mathrm{H}_{2} \mathrm{O}=5.01$ & 5.37 & 5.19 & 5.33 & $5.41 \%$ \\
$\mathrm{~K}_{2} \mathrm{O}=5.71$ & - & - & 5.50 & $5.78 \%$ \\
$\mathrm{As}_{2} \mathrm{O}_{5}=4.65$ & 4.74 & 4.75 & - & $-"$
\end{tabular}

Vorstehende Analysen geben im Mittel $\mathrm{K}_{2} \mathrm{O}: \mathrm{As}_{2} \mathrm{O}_{5}=2.91: 1$, was in Anbetracht des Umstarıdes, dafs die Arsensäurebestimmungen durchweg etwas $z \mathbf{u}$ hoch ausfallen, genügend genau $=3: 1$ ist.

IV. Spaltung def Arsenluteowolframate durch die Karbonate der Alkalien.

1. Spaltung des Ammonsalzes durch Ammonkarbonat.

Ungefähr $30 \mathrm{~g}$ des reinen krystallisierten gelben Ammoniumsalzes wurde in wen ig kaltem Wasser gelöst und tropfenweise eine konzentrierte wässe cige Lösung von Ammonkarbonat hinzugefügt.

${ }^{1}$ Lieb. Ann. $245,54$.

2 2. anorg. Chem: 4, 142. 
Nach Zusatz der ersten Tropfen beginnt eine Ausscheidung glitzernder, weilser Kryställchen, der Menge sich durch erneuten Zusatz stetig vergrölsert, während sich die gelbe Mutterlauge mehr und mehr entfärbt. Sobald dieselbe nahezu farblos geworden, unterbricht man den Prozels, saugt den Niederschlag ab und wäscht mit ganz kleinen Mengen eiskalten Wassers. Die Mutterlauge wurde auf ein bestimmtes Volum gebracht, um darin das Verhältnis der Arsensäure zur Wolframsäure zu bestimmen, und die Krystalle einmal aus Wasser von höchstens $80-90^{\circ}$ umkrystallisiert. Längeres Kochen zerstört die Verbindung, indem sich Ammoniak entwickelt. So wurden dicke, glänzendweilse, halbdurchsichtige Krystalle erhalten, deren Analyse zu der Formel

führte

$$
5\left(\mathrm{NH}_{4}\right)_{2} \mathrm{O} \cdot \mathrm{As}_{2} \mathrm{O}_{5} .17 \mathrm{WO} \mathrm{O}_{3}+8 \mathrm{H}_{2} \mathrm{O}
$$

\footnotetext{
I. $2.3894 \mathrm{~g}$ gaben $1.9968 \mathrm{WO}_{3}$.

II. $2.7508 \mathrm{~g} \# 2.3153 \quad \mathrm{WO}_{\mathrm{8}}$.

III. $2.4536 \mathrm{~g} \quad " \quad 0.1630 \mathrm{Mg}_{2} \mathrm{As}_{2} \mathrm{O}_{7}$ entsprecheud, $0.12093 \mathrm{As}_{9} \mathrm{O}_{5}$.

IV. $2.3468 \mathrm{~g} \quad, \quad 0.1596 \mathrm{Mg}_{2} \mathrm{As}_{2} \mathrm{O}_{7} \quad, \quad, \quad 0.11841 \mathrm{As}_{2} \mathrm{O}_{5}$.
}

Hierans folgt aus I. und III. $\mathrm{As}_{2} \mathrm{O}_{5}: \mathrm{WO}_{3}: 1: 16.80$, "II. " IV. $\mathrm{As}_{2} \mathrm{O}_{5}: \mathrm{WO}_{3}: 1: 16.55$.

Mit Rücksicht auf die Thatsache, dafs die Arsenbestimmung etwas zu hoch ausfällt, erscheint das Verhältnis $\mathrm{As}_{2} \mathrm{O}_{5}: \mathrm{WO}_{3}: 1: 17$ genügend sicher bestimmt.

\section{Bestimmung a Ammoniaks.}

I. $1.0994 \mathrm{~g}$ gaben $0.04128 \mathrm{NH}_{3}$.

IL. $1.3908 \mathrm{~g}, 0.05153 \mathrm{NH}_{3}$.

$$
\begin{array}{ccc}
\text { Berechnet für } & \multicolumn{2}{c}{\text { Gefunden: }} \\
5\left(\mathrm{NH}_{4}\right)_{2} \mathrm{O} \mathrm{As}_{2} \mathrm{O}_{5} .17 \mathrm{WO}_{3}+8 \mathrm{H}_{2} \mathrm{O}: & \mathrm{I} . & \text { II. } \\
\mathrm{NH}_{3}=3.72 \% & \mathrm{NH}=3.75 & 3.71 \%
\end{array}
$$

Da in dem neuen durch Spaltung ents tandenen Ammoniumsalz das Verhältnis zwischen Arsensäure und Wolframsäure zu Gunsten der ersteren geändert ist, so muls in der von der Darstellung herrührenden Mutterlauge die Sache gerade umg ${ }_{\theta}$ kehrt liegen, was die Analyse auch bestätigte. In einem quantitativ durchgeführten Spaltungsversuch wurden aus $28 \mathrm{~g}$ Luteoammonsalz $24 \mathrm{~g}$ des vorstehend beschriebenen Salzes mit $17 \mathrm{WO}_{3}$ und $150 \mathrm{ccm}$ utterlauge erhalten. Letztere wurde in zwei gleiche Teile geteilt, in der einen Hälfte die Wolframsäure und in der zweiten die Arsens zure bestimmt. Es 
ergab sich das Verhältnis $\mathrm{WO}_{3}: \mathrm{As}_{2} \mathrm{O}_{5}=22: 1$. Der verhältnismälsig hohe Gehalt an Arsensäure erklärt sich durch die Löslichkeit des Salzes mit $17 \mathrm{WO}_{3}$. Immerhin ist damit auf einem unabhängigen Wege nochmals bewiesen, dafs der Vorgang eine Spaltung ist, und dals das Hauptprodukt derselben reicher an Arsensäure ist, wie die Stammsubstanz.

\section{Spaltung des Kaliumsalzes durch Kaliumbikarbonat.}

Dieselbe wird gerade so ausgeführt und verläuft ganz analog der Spaltung des Ammonsalzes.

Das K-Salz $5 \mathrm{H}_{2} \mathrm{O} . \mathrm{As}_{2} \mathrm{O}_{5} .17 \mathrm{WO}_{3}+22$ aq bildet nach dem Umkrystallisieren aus siedendem Wasser, wobei es keine Zersetzung erleidet, atlasglänzende, schneeweifse Blättchen, welche in kaltem Wasser kaum löslich sind. Zur Bestimmung des Kaliums diente die Fällung der komplexen Säure mittels Chinolinacetat nach der früher beschriebenen Methode. Wasser und Arsensäure konnten in ein und derselben Portion aus dem Glühverlust und als Magnesiumpyroarseniat bestimmt werden.

I. $1.4324 \mathrm{~g}$ gaben 1.3234 Glühriickstand und darin $0.0898 \mathrm{~g} \mathrm{Mg}_{2} \mathrm{As}_{2} \mathrm{O}_{7}$, entsprechend $0.06663 \mathrm{AsO}_{5}$.

II. $1.6406 \mathrm{~g}$ gaben 1.5140 Glührückstand und darin $0.1012 \mathrm{~g} \mathrm{Mg}_{2} \mathrm{As}_{2} \mathrm{O}_{7}$, entsprechend $0.07508 \mathrm{As}_{2} \mathrm{O}_{5}$.

III. $1.7416 \mathrm{~g}$ wasserhaltiges Salz, entsprechend 1.6078 wasserfreies, gaben $0.2570 \mathrm{~g} \mathrm{KCl}$, entsprechend $0.16213 \mathrm{~K}_{\mathrm{g}} \mathrm{O}$.

IV. $1.1098 \mathrm{~g}$ wasserhaltiges Salz, entsprechend 1.0244 wasserfreies, gaben $0.1656 \mathrm{~g} \mathrm{KCl}$, entsprechend $0.10447 \mathrm{~K}_{2} \mathrm{O}$.

Aus Analyse I. und II. folgt $\mathrm{K}_{2} \mathrm{O}: \mathrm{As}_{2} \mathrm{O}_{5}=4.93: 1$.

",$\quad$ II. " IV. " $\mathrm{K}_{2} \mathrm{O}: \mathrm{As}_{2} \mathrm{O}_{5}=5.10: 1$.

Berechnet für die Formel

$\begin{array}{ccccc}5 \mathrm{~K}_{2} \mathrm{O} . \mathrm{As}_{2} \mathrm{O}_{5} .17 \mathrm{WO}_{3}: & \text { I. } & \text { II. } & \text { III. } & \text { IV. } \\ \mathrm{K}_{2} \mathrm{O}=10.12 \% & - & - & 10.08 & 10.19 \% \\ \mathrm{As}_{2} \mathrm{O}_{5}=4.95 \% & 5.03 & 4.96 & - & -\end{array}$

Berechnet für $22 \mathrm{H}_{2} \mathrm{O}$ :

Gef. Glühverlust im Mittel:

$\mathrm{H}_{2} \mathrm{O}=\mathbf{7 . 8 5} \%$

$\mathrm{H}_{2} \mathrm{O}=7.69 \%$.

Ein wichtiges Ergebnis des Studiums der verschiedenen Phosphorund Arsenwolframsäuren ist die Erfahrung, dals die möglichst gemäfsigte Einwirkung von Alkalikarbonaten auf die sauer reagierenden normalen Salze dieser Säuren regelmälsig zu einer Spaltung des Moleküls führt, indem eine prozentuell nur sehr wenig an Phosphorsäure resp. Arsensäure reichere Salzreihe neben ganz wenig Wol- 
framiat entsteht. Bei oberflächlicher Untersuchung präsentierte sich der betreffende Vorgang ganz wie die Überführung eines sauren Salzes in ein zugehöriges neutrales. Nur eine sehr ins einzelne gehende Erforschung war im stande, den wirklichen Sachverhalt hervortreten zu lassen.

Diese Verhältnisse lassen es jetzt ratsam erscheinen, die von Marignac entdeckten Silikoduodeciwolframiate einem erneuten Studium zu unterwerfen, insbesondere mit Rücksicht darauf, dafs zwei Salzreihen dieser Säure von den resp. Formeln:

existieren sollen.

$$
\begin{aligned}
& 2 \mathrm{R}_{2} \mathrm{O} . \mathrm{SiO}_{2} \cdot 12 \mathrm{WO}_{3}+\mathrm{aq} \text { und } \\
& 4 \mathrm{R}_{2} \mathrm{O} . \mathrm{SiO}_{2} \cdot 12 \mathrm{WO}_{3}+\mathrm{aq}
\end{aligned}
$$

Möglicherweise liegt hier die Sache ebenfalls nicht so einfach, und dals an Basis reichere Salz ist in Wahrheit ein Spaltungsprodukt des anderen von höherem Gehalt an Kieselsäure. Ob diese Vermutung begründet ist, werden darauf hinzielende Versuche ergeben müssen.

Genf, Universitätslaboratorium, August 1899.

Bei der Redaktion eingegangen am 27. September 1899. 